\title{
The effect of supragingival glycine air polishing on periodontitis during maintenance therapy: a randomized controlled trial
}

\author{
Hongye Lu ${ }^{1,2}$, Lu He ${ }^{1,2}$, Yibing Zhao ${ }^{\text {Corresp., }}{ }^{1,2}$, Huanxin Meng ${ }^{\text {Corresp. 1, } 2}$ \\ 1 Department of Periodontology, Peking University School and Hospital of Stomatology, Beijing, China \\ ${ }^{2}$ National Engineering Laboratory for Digital and Material Technology of Stomatology, Beijing Key Laboratory of Digital Stomatology, Beijing, China \\ Corresponding Authors: Yibing Zhao, Huanxin Meng \\ Email address: zhaoyb2000@sina.com, kqhxmeng@bjmu.edu.cn
}

Background. Glycine air polishing has been proved to be safe, comfortable and time-saving. Whether it could substitute ultrasonic scaling to remove dental plaque biofilm during periodontal maintenance remain unclear. The purposes of this study were to evaluate the effect of supragingival glycine air polishing on the subgingival periodontal pathogens during maintenance therapy and to check the association of periodontal pathogens and clinical parameters.

Methods. Twenty-three chronic periodontitis patients during their maintenance therapy were enrolledin the 12-week study. According to randomized split-mouth design, the test side was treated with supragingival glycine $(65 \mu \mathrm{m})$ air polishing (SGAP), while the control side was treated with supragingival ultrasonic scaling and polishing with rubber-cup(SUSP). Clinical examination including plaque index(PLI), probing depth(PD), bleeding index(BI) were performed at baseline and 12 weeks posttreatment.Sampling of the subgingival plaque at each investigational site (mesiobuccal site of the mandibular first molar) was performed at baseline and 2, 4, 8, 12 weeks after maintenance treatment. Four periodontal pathogens including Porphyromonas gingivalis, Tannerella forsythia, Treponema denticola and Fusobacterium nucleatum were detected by 165 rDNA polymerase chain reaction (PCR).

Results. Clinical status generally improved after treatment in both groups. PLI in both groups, PD in SGAP group and bleeding on probing (BOP) (\%) in SUSP group significantly decreased after treatment ( $p$ $<0.05)$. There was no significant difference of clinical parameters between two groups before and after treatment. The detection rates of $P$. gingivalis , $T$. denticola in both groups, $T$. forsythia in SUSP groupand $F$. nucleatum in SGAP group decreased after maintenance treatment in both groups, although no significant difference was found. And it rebound to baseline level at 12 weeks after maintenance treatment. There was no significant difference between SGAP group and SUSP group at any time point. $T$. denticola -positive sites had significantly greater BI than T.denticola -negative sites $(p<0.05)$.

Discussion. Supragingival glycine air polishing had reliable effect in removing subgingival dental plaque biofilm during maintenance period. And three months may be a proper maintenance interval for pockets not more than $5 \mathrm{~mm}$. 
1 The effect of supragingival glycine air polishing on 2 periodontitis during maintenance therapy: a randomized

\section{3 controlled trial}

4 Hongye $\mathrm{Lu}^{1}, \mathrm{Lu} \mathrm{He}^{1}$, Yibing Zhao ${ }^{1 *}$, Huanxin Meng ${ }^{1 *}$

$5 \quad{ }^{1}$ Department of Periodontology, Peking University School and Hospital of Stomatology, National Engineering

6 Laboratory for Digital and Material Technology of Stomatology, Beijing Key Laboratory of Digital Stomatology,

7 Beijing, China

8 Corresponding Author:

9 Huanxin Meng ${ }^{1 *}$, Yibing Zhao ${ }^{1 *}$

$10{ }^{*}$ Two corresponding author contributed equally to this work

11 No. 22, Zhongguancun Nandajie, Haidian District, Beijing, 100081, P.R.China

12 Email address: kqhxmeng@bjmu.edu.cn; zhaoyb2000@sina.com 


\section{Abstract}

14 Background. Glycine air polishing has been proved to be safe, comfortable and time-saving.

\section{Introduction}

42 Periodontitis is the leading factor of tooth loss for adults(Murray et al. 1997; Phipps \& Stevens 43 Whether it could substitute ultrasonic scaling to remove dental plaque biofilm during periodontal maintenance remain unclear. The purposes of this study were to evaluate the effect of supragingival glycine air polishing on the subgingival periodontal pathogens during maintenance therapy and to check the association of periodontal pathogens and clinical parameters.

Methods. Twenty-three chronic periodontitis patients during their maintenance therapy were enrolled in the 12-week study. According to randomized split-mouth design, the test side was treated with supragingival glycine $(65 \mu \mathrm{m})$ air polishing (SGAP), while the control side was treated with supragingival ultrasonic scaling and polishing with rubber-cup(SUSP). Clinical examination including plaque index(PLI), probing depth(PD), bleeding index(BI) were performed at baseline and 12 weeks post-treatment. Sampling of the subgingival plaque at each investigational site (mesiobuccal site of the mandibular first molar) was performed at baseline and 2, 4, 8, 12 weeks after maintenance treatment. Four periodontal pathogens including Porphyromonas gingivalis, Tannerella forsythia, Treponema denticola and Fusobacterium nucleatum were detected by $16 \mathrm{~S}$ rDNA polymerase chain reaction (PCR).

Results. Clinical status generally improved after treatment in both groups. PLI in both groups, PD in SGAP group and bleeding on probing (BOP) (\%) in SUSP group significantly decreased after treatment $(p<0.05)$. There was no significant difference of clinical parameters between two groups before and after treatment. The detection rates of P. gingivalis, $T$. denticola in both groups, T. forsythia in SUSP group and $F$. nucleatum in SGAP group decreased after maintenance treatment in both groups, although no significant difference was found. And it rebound to baseline level at 12 weeks after maintenance treatment. There was no significant difference between SGAP group and SUSP group at any time point. T. denticola-positive sites had significantly greater BI than $T$. denticola-negative sites $(p<0.05)$.

Discussion. Supragingival glycine air polishing had reliable effect in removing subgingival dental plaque biofilm during maintenance period. And three months may be a proper maintenance interval for pockets not more than $5 \mathrm{~mm}$. 1995), and it is initiated by dental plaque biofilm(Gold 1985; Witzel 1882). Therefore, removal of periodontal plaque is of great importance and necessity(Matuliene et al. 2008; Rosling et al. 2001). As patients' self-performed oral hygiene is inadequate to remove the newly formed biofilm, patients with periodontitis who have completed their active periodontal treatment should receive supportive periodontal treatment repeatedly(Axelsson \& Lindhe 1981; Axelsson et al. 2004). Scaling and root planing by hand or ultrasonic instruments is generally adopted to remove biofilm during the maintenance phase(Lindhe \& Nyman 1984). However, it is both timeconsuming and discomfortable, and it may lead to the damage of dentin and cementum to some extent(Petersilka et al. 2003b; Zappa et al. 1991). Air polishing has been accepted to be an effective alternative to remove biofilm and stain (Petersilka et al. 2003c; Zhao et al. 2015), which makes maintenance therapy more time-saving and comfortable. The original material used in air polishing was sodium bicarbonate(Weaks et al. 1984; Willmann et al. 1980), which could cause 
55 severe gingival erosion and substantial root damage(Petersilka et al. 2003a; Simon et al. 2015).

56 Comparing with sodium bicarbonate, glycine is odorless, low-abrasive and highly water-

57 soluble(Bozbay et al. 2016). Furthermore, glycine has been proved to have immunomodulatory,

58 anti-inflammatory and cytoprotective effect for periodontal tissue(Breivik et al. 2005; Schaumann

59 et al. 2013), which makes it an excellent material for periodontal air polishing. Previous study

60 demonstrated that glycine air polishing could save half the treatment time and double the comfort

61 comparing with ultrasonic scaling and polishing with rubber-cup(Zhao et al. 2015).

62 Two main types glycine air polishing are used in the periodontal treatment. One type is 63 supragingival glycine air polishing with standard air-polishing nozzle and $65 \mu \mathrm{m}$ glycine powder;

64 the other type is subgingival glycine air polishing with subgingival nozzle and $25 \mu \mathrm{m}$ glycine 65 powder(Cobb et al. 2017). Several studies have evaluated the clinical and microbiological 66 efficiency of subgingival glycine air polishing. A short-term study compared the effect of 67 subgingival air polishing on 5-8 $\mathrm{mm}$ periodontal pockets in 20 patients with ultrasonic scaling 68 during supportive periodontal therapy(SPT). Bleeding on probing(BOP), probing depth(PD), attachment loss(AL) and bacteria decreased after treatment, but no significant difference between air polishing and ultrasonic scaling was found(Wennstrom et al. 2011). Flemmig et al.(Flemmig et al. 2012) conducted a 90-day study on 30 patients with chronic periodontitis after initial therapy, they applied subgingival air polishing in 4-9mm pockets, and significant reduction was

73 observed in the count of Porphyromonas gingivalis and Tannerella forsythia in moderate-deep 74 pockets. A 12-month randomized controlled trial had evaluated the long-term effect of 75 subgingival air polishing. The number of pockets $>4 \mathrm{~mm}$, PD and BOP significantly reduced at 76 month 12 after treatment. And the prevalence of Aggregatibacter actinomycetemcomitans 77 reduced more in air polishing group than ultrasonic debridement group(Muller et al. 2014). The 78 short-term and long-term efficacy of subgingival air polishing on moderate-to-deep pockets had 79 been confirmed in different studies.

80 Periodontitis during maintenance period should have inflammation well controlled with shallow 81 pockets not more than $5 \mathrm{~mm}$ (Matuliene et al. 2008). Nevertheless, few studies about glycine air 82 polishing focused on this area. Flemmig et al.(Flemmig et al. 2007) applied standard air83 polishing unit in hopeless teeth, then extracted and stained them to assessed the microbiological 84 effect of supragingival air polishing. The morphological result revealed that air polishing 85 debridement efficacy extended up to $5.17 \mathrm{~mm}$ in the subgingival surfaces, and in sites with PD of 863 to $5 \mathrm{~mm}$, the debridement depth reached apically $65 \%-45 \%$ of the periodontal pockets, 87 respectively. It suggested that supragingival glycine air polishing could be used to remove 88 biofilm in pockets not more than $5 \mathrm{~mm}$ during maintenance period. However, very few studies 89 about air polishing involved in this area. Petersilka et al.(Petersilka et al. 2003c; Petersilka et al. 90 2003d) applied supragingival air polishing in $3-5 \mathrm{~mm}$ periodontal pockets and evaluated the 91 subgingival microbiological effect of supragingival air polishing by anaerobe cultivation. 92 Subgingival bacterial counts reduced dramatically by $>99 \%$ after air polishing and subgingival 93 debridement with air polishing was superior to that with curettes. Although previous studies 94 showed satisfying effect of air polishing immediately after treatment, they did not answer how 95 long the microbiological effect would last after glycine air polishing, neither did they evaluate the 96 clinical effect.

97 It is assumed that supragingival air polishing could effectively remove periodontal pathogens in 
98 pockets not more than $5 \mathrm{~mm}$ during maintenance therapy and that the change of periodontal 99 pathogens was in accordance with clinical change. Thus, the objective of the study was to 100 evaluate the follow-up clinical and microbiological effect of supragingival glycine air polishing 101 comparing with ultrasonic scaling and polishing during maintenance phase and to study the 102 relation of periodontal pathogens and clinical changes.

\section{Materials \& Methods}

104 This was an examiner-masked randomized controlled clinical trial with 12-week duration. The 105 split-mouth method was adopted to compare the effect of supragingival glycine air 106 polishing(SGAP) with supragingival ultrasonic scaling and polishing with rubber-cup(SUSP). 107 This study protocol was approved by Biomedical Ethics Committee of the Peking University 108 School and Hospital of Stomatology (approval number: IRB00001052-05106). The clinical study 109 was registered at Chinese Clinical Trial Registry, and the approval number was ChiCTR-INR110 17013073. All participating patients were given information about the study and signed informed 111 consent before the inclusion.

\section{Sample size calculation}

113 According to the microbiological change during maintenance therapy of previous 114 study(Wennstrom et al. 2011), P. gingivalis was the primary outcome and Maximum allowable 115 difference was $20 \%$. Non-inferiority test for two correlated proportions was performed with 116 significant level $\alpha=0.05$ and $\beta=0.2$, and the minimum size was calculated as 20 using software 117 PASS version 11 (NCSS, USA). Considering the rate of possible loss to follow-up 15\%, sample 118 size was set as 23 .

\section{Participants}

120 Twenty-three unrelated Chinese patients with chronic periodontitis were recruited in the study. 121 They all had completed their active treatment and had been maintained for several years at the 122 Department of Periodontology, Peking University School and Hospital of Stomatology, China 123 between August 2011 and March 2013.

124 Inclusion criteria were as follows:

125 - Patients with periodontitis had completed comprehensive periodontal therapy at least three 126 months and were in their maintenance period.

127 Periodontal probing depth not more than $5 \mathrm{~mm}$.

128 Remaining more than 20 teeth

129 Without systemic disease.

130 - No smoking.

131 Exclusion criteria were as follows:

132 Pregnancy or lactation. 
133 Scaling and root planing within three months.

134 - Having periodontal surgery within six months.

135 Antibiotic therapy within three months.

\section{Randomization and interventions}

137 This trial was designed as a randomized split-mouth study. Subject numbers were assigned at the 138 enrollment visit in ascending order. RV.UNIFORM $(0,1)$ was used to generate the randomization 139 table with SPSS v.20.0 (IBM Corp: Armonk, New York, U.S). Then each subject got a random 140 number. According to random number of each subject, the right half-mouth and the left half141 mouth were divided into test or control group, respectively. The test group was conducted with 142 supragingival glycine air polishing(SGAP); while the control group was conducted with 143 supragingival ultrasonic scaling and polishing with rubber-cup and paste(SUSP). Curettes were 144 used to remove the remaining hard calculus if necessary. The intervention was performed by an 145 experienced senior periodontist. Any discomfort or pain during or after treatment should be 146 recorded. The flowchart of the study was showed in Fig. 1.

147 Supragingival air polishing was conducted with $65 \mu \mathrm{m}$ amino acid glycine powder (airflow 148 polishing soft, EMS, Switzerland), Air-Flow handy2(EMS, Switzerland) and Air-Flow Masters 149 (EMS, Switzerland). Ultrasonic scaling was conducted with the use of ultrasonic instrument 150 (Satelec, French), contra-angle handpiece (NSK, Japan), rubber-cup and polishing paste (Ivoclar, 151 Fürstentum Liechtenstein).

\section{Clinical examination}

153 Clinical periodontal examination was performed at baseline before treatment and the endpoint at 15412 weeks. It was carried out by an experienced periodontist, whose self-consistency test showed 155 substantial consistency, with $96 \%$ of the sites differing $1 \mathrm{~mm}$ or less for pocket depth 156 measurements. The examiner was masked with the general information and treatment 157 information of recruited subjects. Criteria for periodontal clinical examination were as follows:

158 (1) Plaque index(PLI): Silness and Löe's(Silness \& Loe 1964)

159 (2) Probing depth(PD): The distance from the bottom of periodontal pockets to gingival margin 160 using Williams probe.

161 (3) Bleeding index(BI): Mazza's(Mazza et al. 1981)

162 (4) Bleeding on probing(BOP): The sites with $\mathrm{BI} \geq 2$ were recorded as BOP-positive.

163 Participants received oral hygiene instruction(OHI) after clinical examination every time.

\section{Sample collection}

165 Samples of the subgingival microbiota at each investigational site was collected at baseline and 2 166 weeks, 4 weeks, 8 weeks and 12 weeks after treatment. Isolated the sampled area, removed the 167 supragingival dental plaque, dried the supragingival tooth surface and collected subgingival 168 dental plaque with sterile curettes in the mesiobuccal sites of two first molars in mandible (if the 169 first molar was lost, the second molar in mandibular would be investigated), and then transferred 170 the samples into sterile Eppendorf tube and stored them in $-80^{\circ} \mathrm{C}$. Sampling was performed 
171 before clinical examination to avoid interacting infection and destruction of plaque by 172 periodontal probing.

\section{DNA extraction and polymerase chain reaction (PCR) measurements}

174 The genomic DNA of bacteria was extracted from subgingival dental plaque. Wash the plaque 175 with $500 \mu \mathrm{l}$ TE buffer $(10 \mathrm{mM}$ Tris-HCL, $1 \mathrm{mM}$ EDTA, pH 7.6) for three times. Add $180 \mu \mathrm{l}$ $17620 \mathrm{mg} / \mathrm{ml}$ lysozyme(Solarbio, China) to the deposit, and water bath at $37^{\circ} \mathrm{C}$ overnight. Microscale 177 genomic DNA extraction kit (TIANGEN, China) was used, according to the manufacturer's 178 instructions. Finally, the concentration and purity of DNA were checked by Nanodrop 179 2000(Thermo Scientific, USA).

180 The samples were analyzed for the detection of four periodontal pathogenic bacteria including 181 P. gingivalis, $T$. forsythia, $T$. denticola, $F$. nucleatum using $16 \mathrm{~S}$ rRNA-based PCR. The DNA 182 extracted from P. gingivalis (ATCC 51700), T. forsythia (ATCC 43037), T. denticola (ATCC 183 33520) and F. nucleatum (ATCC 25586) were used as positive control for PCR analysis, and 184 ultrapure water was used as negative control. The definitive primer followed the reported 185 research of Ashimoto and Baumgartner(Ashimoto et al. 1996; Baumgartner et al. 2004) showed 186 in Table 1.

187 PCR amplification was carried out with GeneAmp PCR system 2700 (ABI, South San Francisco, $188 \mathrm{CA}, \mathrm{USA})$, and the parameters was as follows: PCR mixtures contained $2 \mu$ template, $1 \mu \mathrm{l}$ 189 primer $(10 \mu \mathrm{M})$ primer, $2.5 \mu \mathrm{l} 10 \times$ buffer $\mathrm{Mg}^{2+}$ plus, $2 \mu \mathrm{l} \mathrm{dNTP}(2.5 \mathrm{mM}), 0.2 \mu \mathrm{l}$ Taq DNA 190 polymerase(5U/ul) (TaKaRa Biotechnology, Dalian, PR China), $17.3 \mu \mathrm{ldd} \mathrm{d}_{2} \mathrm{O}$. Thermocycling 191 for P. gingivalis, T. forsythia, T. denticola was $95^{\circ} \mathrm{C} 2 \mathrm{~min} ; 95^{\circ} \mathrm{C} 30 \mathrm{sec}, 60^{\circ} \mathrm{C} 1 \mathrm{~min}, 72^{\circ} \mathrm{C} 1$ $192 \mathrm{~min}, 36$ cycles; $72^{\circ} \mathrm{C} 2 \mathrm{~min}$. Thermocycling for $\mathrm{F}$. nucleatum was $94^{\circ} \mathrm{C} 1 \mathrm{~min} ; 60^{\circ} \mathrm{C} 1 \mathrm{~min}, 72^{\circ} \mathrm{C}$ $19390 \mathrm{sec}, 35$ cycles; $72^{\circ} \mathrm{C} 10 \min 95^{\circ} \mathrm{C} 2 \mathrm{~min} ; 95^{\circ} \mathrm{C} 30 \mathrm{sec}, 60^{\circ} \mathrm{C} 1 \mathrm{~min}, 72^{\circ} \mathrm{C} 1 \mathrm{~min}, 36$ cycles; $19472^{\circ} \mathrm{C} 2 \mathrm{~min}$.

195 PCR products were analyzed by Horizontal Gel Electrophoresis with 1.5\% agarose (Solarbio 196 Beijing, PR China),130V. DL 2000 ladder (TaKaRa Biotechnology, Dalian, PR China) served as 197 the DNA weight marker. The agarose gel was stained with GoldView type I (Solarbio, Beijing, 198 PR China) and taken radiogram with 300nm ultraviolet illumination.

\section{Statistical analysis}

200 The clinical parameters of SGAP group and SUSP were tested for normality with Kolmogorov201 Smirnov method. The parameters following the normal distribution would be tested by paired 202 Student's t-test to compare the difference of two groups. The paired non-parametric test should be 203 applied with Wilcoxon method if variables did not follow normal distribution. Mann-Whitney test 204 was used to compare independent samples which did not follow normal distribution. The clinical 205 parameters of full mouth in SGAP group and SUSP group were accumulated at patient level. The 206 frequency of investigated periodontal pathogens between SGAP group and SUSP group was 207 analyzed by McNemar's test. Longitudinal data was analyzed by McNemar's test for pairwise 208 comparisons. The Spearman rank correlation coefficient was used to determine the rank 209 correlation of clinical parameters and periodontal pathogens. The significance level was preset at 
2100.05 for all statistical tests. SPSS was used to carry out the statistical analyses.

\section{Results}

\section{Subjects}

213 Twenty-two patients, consent to the information, were recruited in the study. They all had initially

214 moderate to severe chronic periodontitis, and they all had regular periodontal maintenance

215 therapy before enrollment. There were 8 males and 14 females, with their age ranging from 28 to

21672 years old. And one patient lost to follow up at week-4 for staying abroad. Totally, eleven

217 subjects' left sides and twelve subjects' right sides were assigned to test group, and all patients

218 were right handed. In all, 290 teeth, 1740 sites in SGAP group and 291 teeth, 1746 sites in SUSP

219 group were clinically examined, and 222 samples (111 in each group) were collected and

220 detected. There was no adverse event during the study period.

\section{Clinical assessment}

222 Clinical parameters of full mouth at individual level in SGAP group and SUSP group were 223 presented in Table 2. The clinical parameters at baseline in SGAP group and SUSP group were 224 undifferentiated, and there were no differences at week 12. All of the clinical parameters declined 225 at week 12 after maintenance treatment in both groups. PLI dramatically dropped from 0.54 and 2260.59 to 0.46 and 0.44 in SGAP group and SUSP group, respectively. And significant difference 227 was observed $(p<0.05)$. PD in SGAP group significantly decreased from $2.48 \mathrm{~mm}$ to $2.34 \mathrm{~mm}(p$ $228<0.05$ ), and the reduction was $0.14 \mathrm{~mm}$. PD in SUSP group decreased from $2.47 \mathrm{~mm}$ to $2.38 \mathrm{~mm}$ 229 and the reduction was $0.09 \mathrm{~mm}$, although no significant difference was found $(p=0.080)$. The BI 230 slightly declined after treatment (SGAP group: from 1.05 to 0.96; SUSP group: from 1.08 to 231 0.97). The percentage of BOP reduced after treatment (SGAP group: from 21.82\% to 18.01\%; 232 SUSP group: from $24.14 \%$ to $17.50 \%$ ), and the change between baseline and week 12 in SUSP 233 group show significant difference $(p<0.05)$.

234 Table 3 displayed the clinical parameters of sampling sites in SGAP group and SUSP group. At 235 baseline, all parameters were homogenous. Sampling sites had mean PD of $2.90 \mathrm{~mm}$ in SGAP 236 group and $3.00 \mathrm{~mm}$ in SUSP group, respectively. At 12 weeks after treatment, the mean PD was $2372.95 \mathrm{~mm}$ in SGAP group and $3.14 \mathrm{~mm}$ in SUSP group, and there was no site with PD more than $2385 \mathrm{~mm}$. No significant difference was found in PD between baseline and 12-week time point. Both 239 groups showed relatively improved periodontal status. Mean BI in SGAP group and SUSP group 240 was respectively 1.32 and 1.27 at baseline, and it became 1.29 and 1.14 at week 12 . A reduction 241 of BI at 12 weeks after treatment was observed in both groups, although no significant difference 242 was found. Supragingival dental plaque measured by PLI significantly decreased in SUSP group $243(p<0.05)$. There was no significant difference between SGAP group and SUSP group in terms of 244 PD, BI and PLI at baseline or 12 weeks.

\section{Microbiological assessments}


246 The detection frequencies of periodontal pathogens in the present study were displayed in Fig. 2. 247 Before treatment, the detection frequencies of $P$. gingivalis, $T$. forsythia, $T$. denticola and $F$. 248 nucleatum between SGAP group and SUSP group had no significant differences. After treatment, 249 a general trend of detection frequency reduction was found in both two groups. However, few 250 significant differences were observed when comparing detection frequencies of different time 251 points within groups. At week 12 the detection frequencies had returned to the level before 252 treatment.

253 Detection frequencies of $P$. gingivalis, $T$. denticola and $F$. nucleatum in both groups declined at 254 week 2 after treatment comparing with baseline (SGAP group: from 26.1\% to 13.0\%; SUSP 255 group: from 34.8\% to 13.0\%), though no significant differences were observed. It kept a relatively 256 low frequency throughout the observation period in SUSP group and rebound subsequently in 257 SGAP group. Regarding T. forsythia, the prevalence did not change much at different time points 258 in SGAP group; while in SUSP group, it decreased from 56.5\% to 34.8\% during the first 2 weeks 259 after treatment, kept a low detection frequency and increased at 12 weeks. Detection frequencies 260 of $T$. denticola dropped off after treatment and then increased at week 4 in both groups. More 261 reduction in SUSP group was observed than that in SGAP group (SGAP group: from 39.1\% to 262 26.1\%; SUSP group: from 30.4\% to 8.7\%). The detection frequency of F. nucleatum did not show 263 change before and after treatment in both groups. At any time point, the two groups didn't show 264 any significant difference in the detection frequencies of all four periodontal pathogens.

265 The comparisons of PD and BI between periodontal pathogen-positive and -negative sites 266 revealed that $T$. denticola exhibited significant difference with BI (Table 4). $T$. denticola- positive 267 sites had significantly greater BI than $T$. denticola-negative sites $(p<0.05)$. Strong association 268 between $T$. denticola and BI was observed $(p<0.05)$.

\section{Discussion}

270 The clinical status had improved 12 weeks after treatment, and the prevalence of periodontal 271 pathogens in subgingival plaque generally decreased after being treated by supragingival air 272 polishing or ultrasonic scaling and polishing. Previous study adopted supragingival glycine air 273 polishing in $3-5 \mathrm{~mm}$ pockets and found that subgingival bacterial counts significantly reduced 274 immediately after air polishing (Petersilka et al. 2003c; Petersilka et al. 2003d). Our study 275 evaluated the microbiological effect of air polishing for longer term with a 12-week follow-up, 276 and the result further confirmed the microbiological effect of supragingival glycine air polishing 277 at 2, 4 and 8 weeks after maintenance therapy. It indicated that supragingival glycine air polishing 278 could remove subgingival periodontal pathogen bacteria in $\leq 5 \mathrm{~mm}$ periodontal pockets 279 effectively.

280 However, air polishing lacks the ability to remove well-mineralized deposit for its low 281 abrasiveness. For patients with firmly attached calculus, ultrasonic scalers or hand instruments 282 are still needed. All the subjects recruited in this study had regular maintenance therapy before 283 enrollment, and only two of them had calculus. The calculus mainly existed in lingual aspect of 284 mandibular anterior teeth, and it was removed by curettes with little effort, and it just cost 285 seconds.

286 Prevalence of periodontal pathogens decreased after treatment and almost rebounded to the pre287 treatment level at 12 weeks, which indicated that dental plaque biofilm could reconstruct within 
28812 weeks. Similar result was found with subgingival air polishing that total count of subgingival 289 bacteria returned to baseline at 90 days after treatment(Flemmig et al. 2012). A randomized 290 controlled trial evaluated the effect of different maintenance recall intervals in patients with 291 chronic periodontitis treated by full-mouth ultrasonic debridement and drew the same conclusion 292 that supportive periodontal therapy at three-month intervals promoted short-term stability of 293 clinical improvements(Ueda et al. 2014). The clinical and microbiological evidence suggested 294 that the interval of air polishing and ultrasonic scaling could be three months during maintenance 295 period. In addition, within this interval, dental plaque would not mineralize into calculus so that it 296 can be removed by air polishing well(White 1997).

297 In present study, no significant differences in clinical parameters and prevalence of four 298 periodontal pathogens between two approaches was observed at any time point. It indicated that 299 supragingival glycine air polishing was equivalent with ultrasonic scaling and polishing with 300 rubber-cup in removing subgingival periodontal pathogens in $\mathrm{PD} \leq 5 \mathrm{~mm}$ sites during maintenance 301 therapy. The comparison between subgingival air polishing and subgingival ultrasonic scaling 302 also drew the same conclusion. A 60-day clinical trial assessed the clinical and microbiological 303 effect of subgingival glycine air polishing comparing subgingival ultrasonic scaling in moderate 304 deep pockets, and it demonstrated no pertinent differences(Wennstrom et al. 2011). The 305 immediate comparison before and after treatment described by Petersilka et al.(Petersilka et al. 306 2003c; Petersilka et al. 2003d) showed that low-abrasive air polishing was superior to curettes in 307 removing subgingival plaque at sites with up to $5 \mathrm{~mm}$ probing depth during maintenance period. 308 Because they adopted sterile paper point to sample, which mainly took unattached plaque. 309 Whereas, scaling and root planing by curettes mainly removed attached plaque. Therefore, their 310 results showed less reduction of bacteria after being treated with curettes.

311 In this study, the prevalence of $P$. gingivalis ranged from $13.0 \%$ to $31.8 \%$ in SGAP group and 312 from $13.0 \%$ to $34.8 \%$ in SUSP group during the observational period. A study in Chinese 313 population showed that the prevalence of $P$. gingivalis in untreated periodontitis was as high as 314 93.0\%(Ding et al. 2010). The prevalence in our study was much lower than that in untreated 315 periodontitis. As the keystone of periodontal pathogens, low prevalence of $P$. gingivalis indicated 316 good infection control(Hajishengallis et al. 2012). A study detected P. gingivalis at 3, 6, 9 and 12 317 months after active periodontal therapy, and the prevalence ranged from 8\%-15\%(Cugini et al. 318 2000). To address this discrepancy, that participants had initially moderate to severe periodontitis 319 before periodontal treatment should be considered.

320 T. denticola was suspected as a major periodontal pathogen which associated with the severity of 321 periodontitis(Takeuchi et al. 2001). Our result demonstrated that T. denticola was significantly 322 associated with BI, which was the most sensitive parameter of periodontal inflammation. Volatile 323 fatty acids, such as succinic acid, acetic acid, propionic acid, butyric acid and isovaleric acid, 324 were found higher in $T$. denticola positive sites than negative sites in aggressive 325 periodontitis(AgP) patients(Lu et al. 2013). Previous study had also proved that PLI, GI (gingiva 326 index), PD, AL were significantly higher in T. denticola positive sites than negative sites(Yuan et 327 al. 2001). The result demonstrated $T$. denticola has close relationship with inflammation.

328 The result of present study displayed that the prevalence of $F$. nucleatum did not change among 329 different time points and persistently maintain a relatively high prevalence. F. nucleatum, as an 330 intermediate colonizer bridging, is one of the initial gram-negative species established in 
331 biofilm(Kolenbrander 2000; Kolenbrander et al. 2002). It could restore to the level before 332 treatment within very short time and exist in subgingival biofilm as a prominent component in 333 quantity.

334 In the study, the spilt-mouth method avoided the interference of complex confounding factors 335 from individual difference. One side usually has similar periodontal status to the other side. 336 Moreover, the stochastic grouping method eliminated the oral hygiene discrepancy from right 337 handedness. Therefore, we could get a reliable result. However, spilt-mouth method also had 338 disadvantage. High-pressure air and glycine particles could make small granules spread to the 339 control side and ultrasonic scaling also has cavitation which have few influence on the other side. 340 However, in consideration that the two sampling sites were far from each other, the impact could 341 almost be neglected.

342 Mandibular first molars were chosen to be sampling sites. Because molars usually suffer more 343 severe periodontitis comparing to incisor and premolar as a result of complex anatomical 344 structure(Jiao et al. 2017). And cleaning first molars thoroughly is important and difficult for both 345 patients and periodontists. If first molar could be effectively treated, other sites would get better 346 therapeutic effect. In addition, the two investigational sites were far from each other that they 347 would not be influenced by the intervention from the other side. Therefore, the clinical and 348 microbiological effect of first molars of mandible were recorded and analyzed.

349 Ultrasonic scaling is the standard of care during maintenance therapy for its effectiveness and 350 efficiency. Comparing with it, supragingival glycine air polishing still has some advantages. Air 351 polishing can be used on both natural teeth and restorative materials, while ultrasonic scaling is 352 restricted to be used on restorative materials for its distorting effect(Vermilyea et al. 1994). 353 Supragingival glycine air polishing is effective in removing stain and it is superior to ultrasonic 354 scaling. Most of patients with periodontitis have exposed root during maintenance period for 355 gingival recession. The application of ultrasonic scaling will leave a rough surface, and that is the 356 reason why additional polishing with rubber-cup is needed; while the damage of glycine air 357 polishing is much smaller. In addition, glycine air polishing can be used in patients who are afraid 358 of the noise of ultrasonic scaling.

359 The safety of glycine air polishing has been reported in several research in vitro and in 360 vivo(Flemmig et al. 2012; Moene et al. 2010; Petersilka et al. 2002), and our study confirmed the 361 safety of supragingival glycine air polishing again. Previous study had proved tooth stain and 362 supragingival biofilm could be removed by supragingival air polishing(Zhao et al. 2015), and 363 present study demonstrated subgingival periodontal pathogens could be removed as well. That is 364 to say, supragingival air polishing could remove exogenous stain, supragingival and subgingival 365 plaque of sites with pockets not more than $5 \mathrm{~mm}$ at the same time(Flemmig et al. 2007; Patil et al. 366 2015; Petersilka et al. 2003c; Petersilka et al. 2003d), which makes it time-saving. The devices of 367 supragingival air polishing and ultrasonic scaling are conventional used and every qualified 368 periodontist and hygienist can operate them well. In addition, supportive periodontal therapy with 369 air polishing is more acceptable and easier to persist on for patients.

370 Considering the advantages and disadvantages of supragingival glycine air polishing, it could be 371 taken as a good alternative to remov1e biofilm of shallow pockets during maintenance period. 372 And not more than $5 \mathrm{~mm}$ pockets during maintenance therapy without much calculus would be 373 the indication for supragingival air polishing use. Further study is needed to compare the clinical 
374 and microbiological effect for longer term.

\section{Conclusion}

376 Supragingival glycine air polishing had reliable effect in improving clinical status and removing 377 subgingival periodontal pathogens during maintenance therapy, and the effect was similar to 378 ultrasonic scaling and polishing with rubber-cup. Three months may be a proper maintenance 379 interval for pockets not more than $5 \mathrm{~mm}$.

380 Acknowledgments

381 We especially thank Zhibin Chen, Xianghui Feng, Jian Jiao and Wenli Song (Department of 382 Periodontology, Peking University School and Hospital of Stomatology, China) for their generous 383 help.

384

385

386

387

388

389

390

391

392

393

394

395

396

397

398

399

400

401

402

403

404

405

406

407

408

409

410

411

412

413

414

\section{Reference}

Ashimoto A, Chen C, Bakker I, and Slots J. 1996. Polymerase chain reaction detection of 8 putative periodontal pathogens in subgingival plaque of gingivitis and advanced periodontitis lesions. Oral Microbiol Immunol 11:266-273.

Axelsson P, and Lindhe J. 1981. The significance of maintenance care in the treatment of periodontal disease. J Clin Periodontol 8:281-294.

Axelsson P, Nystrom B, and Lindhe J. 2004. The long-term effect of a plaque control program on tooth mortality, caries and periodontal disease in adults. Results after 30 years of maintenance. J Clin Periodontol 31:749-757. 10.1111/j.1600-051X.2004.00563.x

Baumgartner JC, Siqueira JF, Jr., Xia T, and Rocas IN. 2004. Geographical differences in bacteria detected in endodontic infections using polymerase chain reaction. J Endod 30:141-144. 10.1097/00004770-200403000-00004

Bozbay E, Dominici F, Gokbuget AY, Cintan S, Guida L, Aydin MS, Mariotti A, and Pilloni A. 2016. Preservation of root cementum: a comparative evaluation of power-driven versus hand instruments. Int J Dent Hyg. 10.1111/idh.12249

Breivik T, Gundersen Y, Fonnum F, Vaagenes P, and Opstad PK. 2005. Chronic glycine treatment inhibits ligature-induced periodontal disease in Wistar rats. J Periodontal Res 40:43-47. 10.1111/j.1600-0765.2004.00767.x

Cobb CM, Daubert DM, Davis K, Deming J, Flemmig TF, Pattison A, Roulet JF, and Stambaugh RV. 2017. Consensus Conference Findings on Supragingival and Subgingival Air Polishing. Compend Contin Educ Dent 38:e1-e4.

Cugini MA, Haffajee AD, Smith C, Kent RL, Jr., and Socransky SS. 2000. The effect of scaling and root planing on the clinical and microbiological parameters of periodontal diseases: 12-month results. J Clin Periodontol 27:30-36.

Ding F, Meng HX, Li QQ, Zhao YB, Feng XH, and Zhang L. 2010. [Effect of periodontal mechanical treatment on periodontal pathogenic bacteria in gingival crevicular fluid of chronic periodontitis patients]. Beijing Da Xue Xue Bao 42:202-206.

Flemmig TF, Arushanov D, Daubert D, Rothen M, Mueller G, and Leroux BG. 2012. Randomized controlled trial assessing efficacy and safety of glycine powder air polishing in moderate-to-deep periodontal pockets. J Periodontol 83:444-452. 10.1902/jop.2011.110367 
415

416

417

418

419

420

421

422

423

424

425

426

427

428

429

430

431

432

433

434

435

436

437

438

439

440

441

442

443

444

445

446

447

448

449

450

451

452

453

454

455

456

457

Flemmig TF, Hetzel M, Topoll H, Gerss J, Haeberlein I, and Petersilka G. 2007. Subgingival debridement efficacy of glycine powder air polishing. J Periodontol 78:1002-1010. 10.1902/jop.2007.060420

Gold SI. 1985. Paeeriodontics. The past. Microbiology. Part (III). J Clin Periodontol 12:257-269. Hajishengallis G, Darveau RP, and Curtis MA. 2012. The keystone-pathogen hypothesis. Nat Rev Microbiol 10:717-725. 10.1038/nrmicro2873

Jiao J, Shi D, Cao ZQ, Meng HX, Lu RF, Zhang L, Song Y, and Zhao JR. 2017. Effectiveness of non-surgical periodontal therapy in a large Chinese population with chronic periodontitis. J Clin Periodontol 44:42-50. 10.1111/jcpe.12637

Kolenbrander PE. 2000. Oral microbial communities: biofilms, interactions, and genetic systems. Annu Rev Microbiol 54:413-437. 10.1146/annurev.micro.54.1.413

Kolenbrander PE, Andersen RN, Blehert DS, Egland PG, Foster JS, and Palmer RJ, Jr. 2002. Communication among oral bacteria. Microbiol Mol Biol Rev 66:486-505, table of contents.

Lindhe J, and Nyman S. 1984. Long-term maintenance of patients treated for advanced periodontal disease. J Clin Periodontol 11:504-514.

Lu RF, Feng L, Gao XJ, Meng HX, and Feng XH. 2013. [Relationship between volatile fatty acids and Porphyromonas gingivalis and Treponema denticola in gingival crevicular fluids of patients with aggressive periodontitis]. Beijing Da Xue Xue Bao 45:12-16.

Matuliene G, Pjetursson BE, Salvi GE, Schmidlin K, Bragger U, Zwahlen M, and Lang NP. 2008. Influence of residual pockets on progression of periodontitis and tooth loss: results after 11 years of maintenance. J Clin Periodontol 35:685-695. 10.1111/j.1600051X.2008.01245.x

Mazza JE, Newman MG, and Sims TN. 1981. Clinical and antimicrobial effect of stannous fluoride on periodontitis. J Clin Periodontol 8:203-212.

Moene R, Decaillet F, Andersen E, and Mombelli A. 2010. Subgingival plaque removal using a new air-polishing device. J Periodontol 81:79-88. 10.1902/jop.2009.090394

Muller N, Moene R, Cancela JA, and Mombelli A. 2014. Subgingival air-polishing with erythritol during periodontal maintenance: randomized clinical trial of twelve months. J Clin Periodontol 41:883-889. 10.1111/jcpe.12289

Murray H, Clarke M, Locker D, and Kay EJ. 1997. Reasons for tooth extractions in dental practices in Ontario, Canada according to tooth type. Int Dent J 47:3-8.

Patil SS, Rakhewar PS, Limaye PS, and Chaudhari NP. 2015. A comparative evaluation of plaque-removing efficacy of air polishing and rubber-cup, bristle brush with paste polishing on oral hygiene status: A clinical study. J Int Soc Prev Community Dent 5:457-462. 10.4103/2231-0762.167723

Petersilka GJ, Bell M, Mehl A, Hickel R, and Flemmig TF. 2003a. Root defects following air polishing. J Clin Periodontol 30:165-170.

Petersilka GJ, Draenert M, Jervoe-Storm PM, Heinecke A, and Flemmig TF. 2002. Assessment of root curvature and distance using computed tomography. Clin Oral Investig 6:171174. 10.1007/s00784-002-0165-4

Petersilka GJ, Draenert M, Mehl A, Hickel R, and Flemmig TF. 2003b. Safety and efficiency of novel sonic scaler tips in vitro. J Clin Periodontol 30:551-555. 
458

459

460

461

462

463

464

465

466

467

468

469

470

471

472

473

474

475

476

477

478

479

480

481

482

483

484

485

486

487

488

489

490

491

492

493

494

495

496

497

498

499

500
Petersilka GJ, Steinmann D, Haberlein I, Heinecke A, and Flemmig TF. 2003c. Subgingival plaque removal in buccal and lingual sites using a novel low abrasive air-polishing powder. J Clin Periodontol 30:328-333.

Petersilka GJ, Tunkel J, Barakos K, Heinecke A, Haberlein I, and Flemmig TF. 2003d. Subgingival plaque removal at interdental sites using a low-abrasive air polishing powder. J Periodontol 74:307-311. 10.1902/jop.2003.74.3.307

Phipps KR, and Stevens VJ. 1995. Relative contribution of caries and periodontal disease in adult tooth loss for an HMO dental population. J Public Health Dent 55:250-252.

Rosling B, Serino G, Hellstrom MK, Socransky SS, and Lindhe J. 2001. Longitudinal periodontal tissue alterations during supportive therapy. Findings from subjects with normal and high susceptibility to periodontal disease. J Clin Periodontol 28:241-249.

Schaumann T, Kraus D, Winter J, Wolf M, Deschner J, and Jager A. 2013. Potential immune modularly role of glycine in oral gingival inflammation. Clin Dev Immunol 2013:808367. 10.1155/2013/808367

Silness J, and Loe H. 1964. PERIODONTAL DISEASE IN PREGNANCY. II. CORRELATION BETWEEN ORAL HYGIENE AND PERIODONTAL CONDTION. Acta Odontol Scand 22:121135.

Simon CJ, Munivenkatappa Lakshmaiah Venkatesh P, and Chickanna R. 2015. Efficacy of glycine powder air polishing in comparison with sodium bicarbonate air polishing and ultrasonic scaling - a double-blind clinico-histopathologic study. Int J Dent Hyg 13:177183. 10.1111 /idh.12133

Takeuchi Y, Umeda M, Sakamoto M, Benno Y, Huang Y, and Ishikawa I. 2001. Treponema socranskii, Treponema denticola, and Porphyromonas gingivalis are associated with severity of periodontal tissue destruction. J Periodontol 72:1354-1363. 10.1902/jop.2001.72.10.1354

Ueda PH, Casati MZ, Casarin RC, Pera C, Pimentel SP, and Cirano FR. 2014. Supportive periodontal treatment and full-mouth ultrasonic debridement: a randomised controlled clinical trial. Oral Health Prev Dent 12:323-329. 10.3290/j.ohpd.a31664

Vermilyea SG, Prasanna MK, and Agar JR. 1994. Effect of ultrasonic cleaning and air polishing on porcelain labial margin restorations. J Prosthet Dent 71:447-452.

Weaks LM, Lescher NB, Barnes CM, and Holroyd SV. 1984. Clinical evaluation of the ProphyJet as an instrument for routine removal of tooth stain and plaque. J Periodontol 55:486-488. 10.1902/jop.1984.55.8.486

Wennstrom JL, Dahlen G, and Ramberg P. 2011. Subgingival debridement of periodontal pockets by air polishing in comparison with ultrasonic instrumentation during maintenance therapy. J Clin Periodontol 38:820-827. 10.1111/j.1600051X.2011.01751.x

White DJ. 1997. Dental calculus: recent insights into occurrence, formation, prevention, removal and oral health effects of supragingival and subgingival deposits. Eur J Oral Sci 105:508-522.

Willmann DE, Norling BK, and Johnson WN. 1980. A new prophylaxis instrument: effect on enamel alterations. J Am Dent Assoc 101:923-925.

Witzel A. 1882. The treatment of pyorrhea alveolaris or infectious alveolitis. BrJ Dent Sci 
$501 \quad 25: 153$

502 Yuan K, Chang CJ, Hsu PC, Sun HS, Tseng CC, and Wang JR. 2001. Detection of putative 503 periodontal pathogens in non-insulin-dependent diabetes mellitus and non-diabetes 504 mellitus by polymerase chain reaction. J Periodontal Res 36:18-24.

505 Zappa U, Smith B, Simona C, Graf H, Case D, and Kim W. 1991. Root substance removal by 506 scaling and root planing. J Periodontol 62:750-754. 10.1902/jop.1991.62.12.750

507 Zhao $\mathrm{Y}, \mathrm{He} \mathrm{L}$, and Meng H. 2015. [Clinical observation of glycine powder air-polishing during 508 periodontal maintenance phase]. Zhonghua Kou Qiang Yi Xue Za Zhi 50:544-547. 


\section{Table $\mathbf{1}$ (on next page)}

Primers used in PCR analysis 
1 Table 1. Primers used in PCR analysis

\begin{tabular}{|c|c|c|c|}
\hline & Primer sequence( $\left.5^{\prime}-3^{\prime}\right)$ & $\begin{array}{l}\text { Base position } \\
\text { (length) }\end{array}$ & References \\
\hline \multicolumn{4}{|c|}{ Porphyromonas gingivalis } \\
\hline Forward & AGG CAG CTT GCC ATA CTG CG & \multirow[t]{2}{*}{$729-1,132(404)$} & \multirow[t]{2}{*}{ Ashimoto et al. } \\
\hline Reverse & ACT GTT AGC AAC TAC CGA TGT & & \\
\hline \multicolumn{4}{|c|}{ Tannerella forsythia } \\
\hline Forward & GCG TAT GTA ACC TGC CCG CA & \multirow[t]{2}{*}{$120-760(641)$} & \multirow[t]{2}{*}{ Ashimoto et al. } \\
\hline Reverse & TGC TTC AGT GTC AGT TAT ACC T & & \\
\hline \multicolumn{4}{|c|}{ Treponema denticola } \\
\hline Forward & $\begin{array}{l}\text { TAA TAC CGA ATG TGC TCA TTT } \\
\text { ACA T }\end{array}$ & \multirow[t]{2}{*}{$193-508$ (316) } & \multirow[t]{2}{*}{ Ashimoto et al. } \\
\hline Reverse & $\begin{array}{l}\text { TCA AAG AAG CAT TCC CTC TTC } \\
\text { TTC TTA }\end{array}$ & & \\
\hline \multicolumn{4}{|c|}{ Fusobacterium nucleatum } \\
\hline Forward & AGGGCATCCTAGAATTATG & \multirow[t]{2}{*}{$190-1,006(817)$} & \multirow[t]{2}{*}{ Baumgartner et al. } \\
\hline Reverse & GGGACACTGAAACATCTCTGTCTCA & & \\
\hline
\end{tabular}

2 


\section{Figure 1 (on next page)}

Flow diagram of the progress through the phases of the randonmized trial.

SGAP group, supragingival glycine air polishing group; SUSP group, supragingival ultrasonic scaling group. 


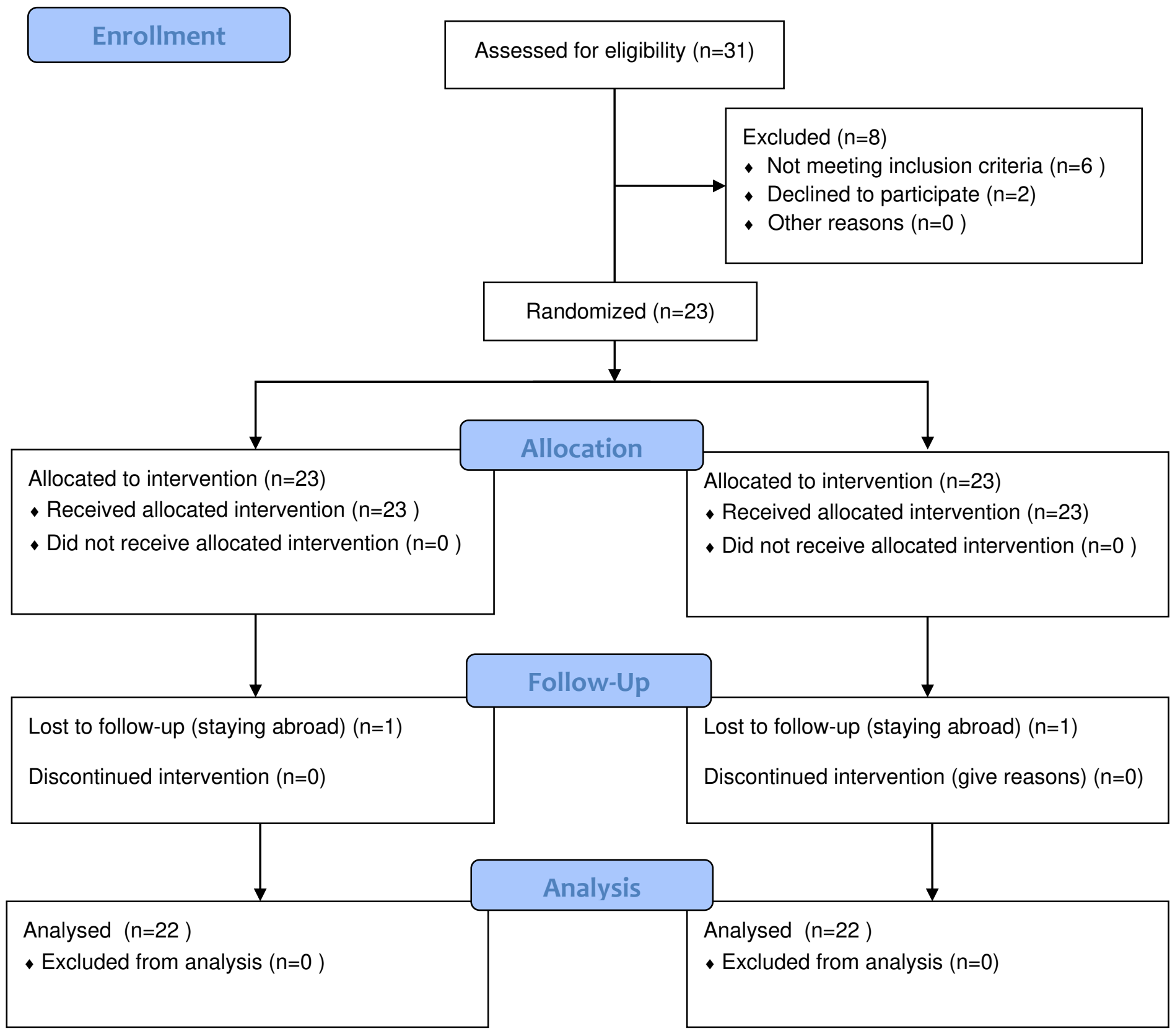




\section{Table 2 (on next page)}

Clinical parameters of all investigational sites in SGAP group and SUSP group.

SGAP group, supragingival glycine air polishing group; SUSP group, supragingival ultrasonic scaling group; PLI: plaque index; PD, probing depth; $\mathrm{Bl}$, bleeding index; BOP, bleeding on probing.

*Significant difference between baseline and 12 week. 
1 Table 2. Clinical parameters of all investigational sites in SGAP group and SUSP group $2 \quad($ mean $\pm \mathrm{SD})$

\begin{tabular}{lccc}
\hline \multicolumn{2}{c}{ SGAP group $(\mathrm{n}=22)$} & SUSP group $(\mathrm{n}=22)$ & $\boldsymbol{p}$ Value \\
\hline PLI & & & \\
Baseline & $0.54 \pm 0.25^{*}$ & $0.59 \pm 0.33^{*}$ & 0.267 \\
$\mathbf{1 2}$ weeks & $0.46 \pm 0.34^{*}$ & $0.44 \pm 0.24^{*}$ & 0.733 \\
Reduction & $0.08 \pm 0.33$ & $0.15 \pm 0.17$ & 0.230 \\
$\boldsymbol{p}$ Value & $\mathbf{0 . 0 4 6}$ & $\mathbf{0 . 0 0 0}$ & \\
PD (mm) & & & \\
Baseline & $2.48 \pm 0.32^{*}$ & $2.47 \pm 0.30$ & 0.693 \\
$\mathbf{1 2}$ weeks & $2.34 \pm 0.32^{*}$ & $2.38 \pm 0.27$ & 0.291 \\
Reduction & $0.14 \pm 0.23$ & $0.09 \pm 0.23$ & 0.091 \\
$\boldsymbol{p}$ Value & $\mathbf{0 . 0 1 3}$ & 0.080 & \\
BI & & & 0.547 \\
Baseline & $1.05 \pm 0.31$ & $1.08 \pm 0.37$ & 0.961 \\
12 weeks & $0.96 \pm 0.29$ & $0.97 \pm 0.26$ & 0.745 \\
Reduction & $0.11 \pm 0.36$ & $0.11 \pm 0.41$ & \\
$\boldsymbol{p}$ Value & 0.249 & 0.230 & \\
BOP (\%) & & & 0.368 \\
Baseline & $21.82 \pm 11.76$ & $24.14 \pm 13.32^{*}$ & 0.315 \\
$\mathbf{1 2}$ weeks & $18.01 \pm 11.38$ & $17.50 \pm 10.35^{*}$ & 0.334 \\
Reduction & $3.81 \pm 10.6$ & $6.64 \pm 12.04$ & $\mathbf{0 . 0 1 7}$ \\
$\boldsymbol{p}$ Value & 0.107 & & \\
\hline
\end{tabular}

3 SGAP group, supragingival glycine air polishing group; SUSP group, supragingival ultrasonic scaling and polishing

4 group; PLI: plaque index; PD, probing depth; BI, bleeding index; BOP, bleeding on probing.

$5 *$ Significant difference between baseline and 12 weeks. 


\section{Table 3(on next page)}

Clinical parameters of sampling sites in SGAP group and SUSP group.

SGAP group, supragingival glycine air polishing group; SUSP group, supragingival ultrasonic scaling group; PLI: plaque index; PD, probing depth; BI, bleeding index.

*Significant difference between baseline and 12 weeks. 
1 Table 3, Clinical parameters of sampling sites in SGAP group and SUSP group (mean \pm SD)

\begin{tabular}{lrrr}
\hline \multicolumn{2}{c}{ SGAP group $(\mathrm{n}=22)$} & SUSP group $(\mathrm{n}=22)$ & $\boldsymbol{p}$ Value \\
\hline PLI & & & \\
Baseline & $0.36 \pm 0.49$ & $0.54 \pm 0.51^{*}$ & 0.366 \\
$\mathbf{1 2}$ weeks & $0.50 \pm 0.74$ & $0.27 \pm 0.16^{*}$ & 0.194 \\
$\boldsymbol{p}$ Value & 0.439 & $\mathbf{0 . 0 1 4}$ & \\
PD (mm) & & & \\
Baseline & $2.91 \pm 0.87$ & $3.00 \pm 0.87$ & 0.917 \\
$\mathbf{1 2}$ weeks & $2.95 \pm 0.90$ & $3.14 \pm 0.47$ & 0.506 \\
$\boldsymbol{p}$ Value & 0.862 & 0.334 & \\
BI & & & 0.854 \\
Baseline & $1.32 \pm 0.84$ & $1.27 \pm 0.70$ & 0.536 \\
$\mathbf{1 2}$ weeks & $1.23 \pm 0.69$ & $1.09 \pm 0.81$ & \\
$\boldsymbol{p}$ Value & 0.672 & 0.248 & \\
\hline
\end{tabular}

2 SGAP group, supragingival glycine air polishing group; SUSP group, supragingival ultrasonic scaling and polishing

3 group; PLI: plaque index; PD, probing depth; BI, bleeding index.

4 *Significant difference between baseline and 12 weeks. 


\section{Figure 2 (on next page)}

The prevalence of four periodontal pathogens at different time point.

SGAP group, supragingival glycine air polishing group; SUSP group, supragingival ultrasonic scaling group. 


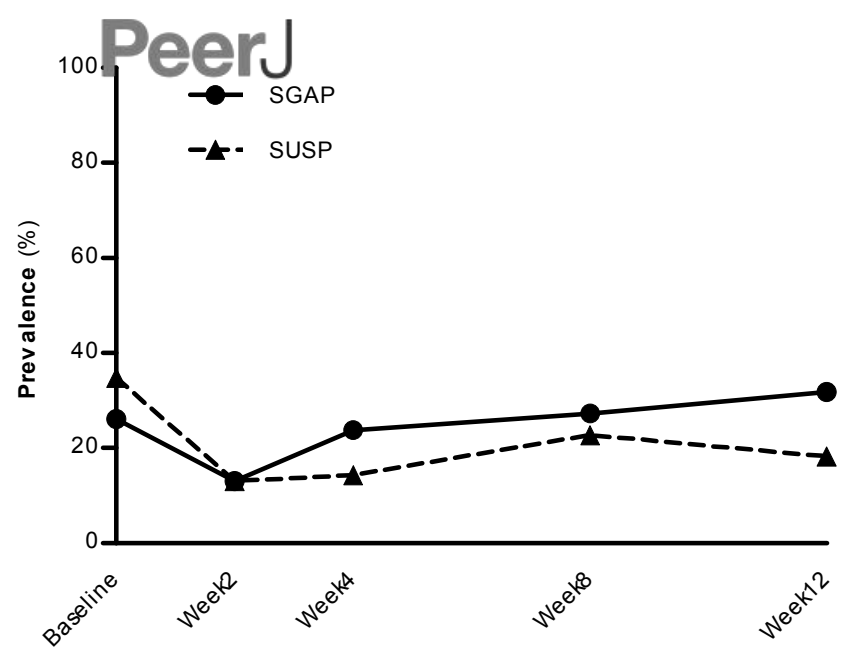

(A) P. gingivalis

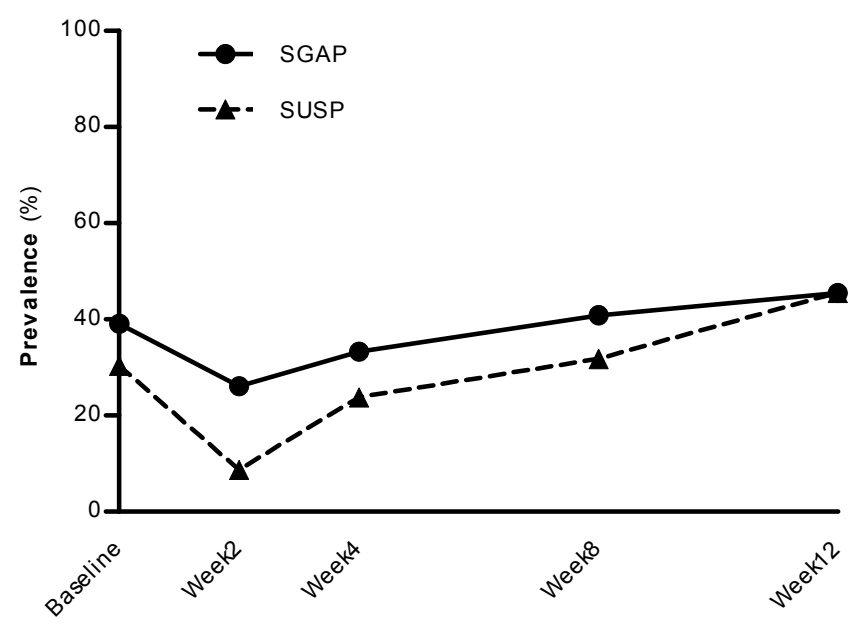

(C) T. denticola

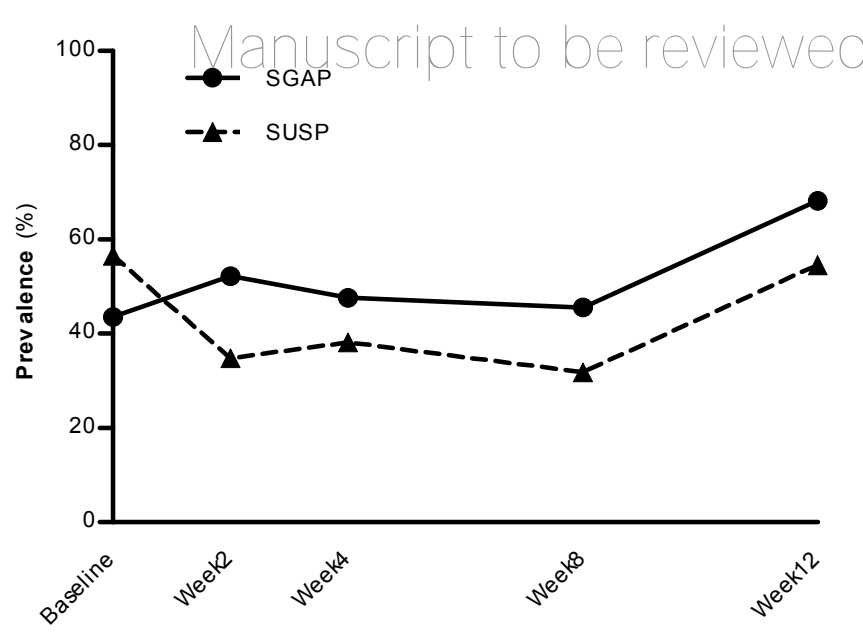

(B) T. forsythia

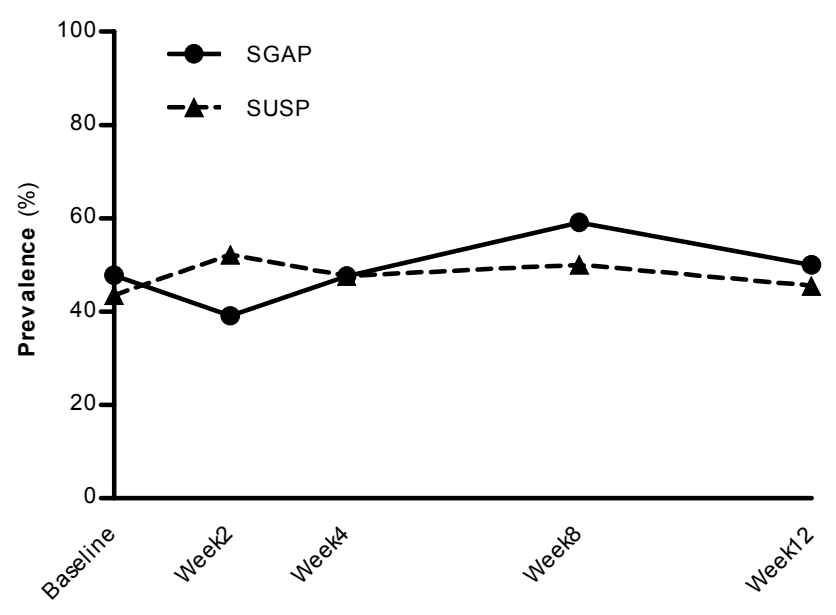

(D) F. nucleatum 


\section{Table 4 (on next page)}

Comparison of PD and BI between periodontal pathogens-positive and -negative sites.

SGAP group, supragingival glycine air polishing group; SUSP group, supragingival ultrasonic scaling group; PLI: plaque index; PD, probing depth; BI, bleeding index.

*Significant difference between baseline and 12 weeks. 
1 Table 4. Comparison of PLI, PD and BI between periodontal pathogens-positive and -negative

2 sites (mean $\pm \mathrm{SD})$

\begin{tabular}{lrrr}
\hline & \multicolumn{1}{c}{ PLI } & PD $(\mathrm{mm})$ & \multicolumn{1}{c}{ BI } \\
\hline P. gingivalis & & & \\
Positive & $0.60 \pm 0.71$ & $2.96 \pm 0.61$ & $1.44 \pm 0.71$ \\
Negative & $0.35 \pm 0.48$ & $2.95 \pm 0.93$ & $1.17 \pm 0.74$ \\
$p$ Value & 0.148 & 0.939 & 0.150 \\
$\boldsymbol{T}$. forsythia & & & \\
Positive & $0.46 \pm 0.59$ & $2.92 \pm 0.83$ & $1.32 \pm 0.65$ \\
Negative & $0.38 \pm 0.54$ & $3.00 \pm 0.88$ & $1.15 \pm 0.83$ \\
$p$ Value & 0.484 & 0.586 & 0.286 \\
$\boldsymbol{T}$ denticola & & & \\
Positive & $0.39 \pm 0.49$ & $3.11 \pm 0.82$ & $1.47 \pm 0.65^{*}$ \\
Negative & $0.44 \pm 0.60$ & $2.85 \pm 0.86$ & $1.09 \pm 0.76^{*}$ \\
$p$ Value & 0.839 & 0.107 & $\mathbf{0 . 0 3 0}$ \\
$\boldsymbol{F}$. nucleatum & & & \\
Positive & $0.43 \pm 0.55$ & $2.90 \pm 0.69$ & $1.28 \pm 0.74$ \\
Negative & $0.42 \pm 0.58$ & $3.00 \pm 0.97$ & $1.21 \pm 0.74$ \\
$p$ Value & 0.835 & 0.558 & 0.803 \\
\hline
\end{tabular}

3 PLI, plaque index; PD, probing depth; BI, bleeding index.

$4 *$ Statistically significant differences between positive- and negative-sites. 